ИЗВЕСТИЯ АКАДЕМИИ НАУК ЭСТОНСКОП ССР. ФИЗИКА * МАТЕМАТИКА PROCEEDINGS OF THE ACADEMY OF SCIENCES OF THE ESTONIAN SSR. PHYSICS * MATHEMATICS

$1988,37,4$

удК $535.343 .2 ; 538.958$

A. ШЕРМАH

\title{
О МАТРИЧНОМ РЕКУРСИВНОМ МЕТОДЕ ВЫЧИСЛЕНИЯ ФУНКЦИЙ ГРИНА
}

\author{
(Представил В. Хиюснков)
}

Рекурсивные методы. вычисления функций Грина (ФГ) являются эффективным средством решения широкого круга квантово-механических задач $\left[{ }^{1-4}\right]$. Различают обычный (PM) и матричный (MPM) peкурсивные методы. Последний, являясь некоторым обобщением РМ, применяется, как правило, для лучшего учета некоторых симметрийных особенностей гамильтониана задачи $\left[{ }^{1,2}\right]$. Целью данного сообщения является обсуждение некоторых особенностей MPM, делающих этот метод в ряде случаев более эффективным в сравнении с РМ. Эти особенности состоят в следующем. I. При неизбежном в процессе счета ограничении базиса состояний MPM в предлагаемой ниже формулировке описывает значительно большее количество полюсов, т. е. дает более полную информацию о ФГ, чем РМ. Для описания разрезов и точек ветвления ФГ в РМ приходится использовать различные виды экстраполяции последующих элементов цепной дроби, описывающей эту функцию, исходя из вычисленных (см., напр., $\left.\left[{ }^{1-4}\right]\right)$. Слабо влияя на наиболее существенные особенности ФГ, эта экстраполяция может заметно искажать более слабые детали [ $\left.{ }^{3}\right]$. Бо́льшая информативность MPM позволяет в принципе избавиться от таких труднопроверяемых приближений. II. В предлагаемой формулировке MPM удается избежать и другого недостатка РM - неустойчивости алгоритма, связанной с потерей ортогональности векторов базиса Ланцоша [5]. За указанные преимущества в MPM приходится расплачиваться некоторым усложнением вычислений: как следует из названия, основные уравнения имеют в общем случае матричный вид.

Начнем со случая, когда в ФГ усреднение проводится по некоторому чистому состоянию, которое обозначим вектором $|0,1\rangle$.; ФГ имеет вид $G_{11}^{0}(z)=\left\langle 0,1\left|(z-H)^{-1}\right| 0,1\right\rangle$, где $H=H^{+}-$гамильтониан рассматриваемой системы. Подействуем гамильтонианом на $|0,1\rangle$. Полученный вектор можно представить как линейную комбинацию вектора $|0,1\rangle$ и ряда других векторов, вид которых зависит от избранного базиса (куда входит и вектор $|0,1\rangle$. Выбор базиса диктуется характером задачи; при удачном выборе коэффициенты в этой линейной комбинации легко определяются (см. приведенный ниже пример), в некоторых случаях - непосредственно из вида гамильтониана. Будем далее считать, что векторы базиса ортонормированы (от этого предположения, в принципе, можно отказаться). Обозначим фигурирующие в линейной комбинации базисные векторы (исключая $|0,1\rangle$ ) $|1, \alpha\rangle$. Действуя на один из этих векторов гамильтонианом, получаем линейную комбинацию, содержащую векторы $|0,1\rangle,|1, \alpha\rangle$ и некоторые новые векторы, которые обозначим $|2, \beta\rangle$. Продолжая эту процедуру, заметим, что ввиду эрмитовости гамильтониана в линейную комбинацию $H|n, \alpha\rangle, n>1$, входят лишь векторы $|n+1, \beta\rangle,|n, \gamma\rangle$ и $|n-1, \delta\rangle$. Действительно, поскольку по построению $\langle n, \alpha|H| n-k, \beta\rangle=$ 
$=0, k \geqslant 2$, то $\langle n-k, \beta|H| n, \alpha\rangle=0$. Таким образом, в подпространствё полного гильбертова пространства, определяемом вектором $|0,1\rangle$, гамильтониан представляется в блок-трехдиагональном виде

$$
H=\sum_{n=0}^{\infty} \sum_{\alpha, \beta}\left[|n, \alpha\rangle E_{\alpha \beta}^{n}\langle n, \beta|+\left(|n, \alpha\rangle V_{\alpha \beta}^{n+1}\langle n+1, \beta|+\text { h. c. }\right)\right] \text {. }
$$

Описанная выше процедура является, по существу, матричным обобщением известного алгоритма Ланцоша $[1,2,5]$ с тем, однако, весьма важным отличием, что в (1) учтена структура гамильтониана в данном базисе, тогда как в алгоритме Ланцоша вне зависимости от вида гамильтониан всегда сводится к гамильтониану полубесконечной цепи с парным взаимодействием или какого-либо ее векторного обобщения, лишь отчасти учитывающего эту структуру. Это различие двух подходов приводит к существенному различию в тех усилиях, которые нужно затратить на определение коэффициентов $E_{\alpha \beta}^{n}$ и $V_{\alpha \beta}^{n}$ в (1) и аналогичных величин в алгоритме Ланцоша: как уже отмечалось, первые находятся сравнительно просто, в некоторых случаях без каких-либо вычислений, тогда как задача определения вторых - наиболее сложная и трудоемкая часть РM (см., напр., $\left.\left[{ }^{1-4}\right]\right)$.

Коэффициенты $E_{\alpha \beta}^{n}$ и $V_{\alpha \beta}^{n}$, а также число векторов с номером $n$ изменяются при переходе к иному базису. Подчеркнем, что наличие малых параметров в гамильтониане позволяет существенно упростить задачу вычисления ФГ подходящим выбором базиса.

Если вектор $|0,1\rangle$ под действием операций симметрии преобразуется в соответствии с определенным неприводимым представлением группы симметрии гамильтониана, остальные векторы $|n, \alpha\rangle$ можно и в некоторых случаях предпочтительно выбрать таким образом, чтобы они также преобразовывались по той же строке того же неприводимого представления. Такая симметризация ведет к сокращению числа операций и объема требуемой памяти ЭВМ при вычислении $Ф Г$; с другой стороны, она может несколько усложнить процедуру нахождения коэффициентов $E_{\alpha \beta}^{n}$ и $V_{\alpha \beta}^{n}$.

Введем обозначения

$$
\begin{aligned}
& H_{n}=H_{0}+H_{i n}=\sum_{m=0}^{\infty} \sum_{\alpha, \beta}|m, \alpha\rangle E_{\alpha \beta}^{m}\langle m, \beta|+ \\
& +\sum_{m=n}^{\infty} \sum_{\alpha, \beta}\left(|m, \alpha\rangle V_{\alpha \beta}^{m+1}\langle m+1, \beta|+\text { h. c. }\right), \\
& G_{\alpha \beta}^{n}(z)=\left\langle n, \alpha\left|\left(z-H_{n}\right)^{-1}\right| n, \beta\right\rangle, \quad g_{\alpha \beta}^{n}(z)=\left\langle n, \alpha\left|\left(z-H_{0}\right)^{-1}\right| n, \beta\right\rangle, \\
& \Lambda_{n}=\prod_{\alpha}(1-|n, \alpha\rangle\langle n, \alpha|)=1-\sum_{\alpha}|n, \alpha\rangle\langle n, \alpha| .
\end{aligned}
$$

Заметим, что

$$
\begin{gathered}
\left(z-H_{n}\right)^{-1} \Lambda_{n}=\Lambda_{n}\left(z-H_{n+1}\right)^{-1} \Lambda_{n}+ \\
+\left(z-H_{n}\right)^{-1}\left(1-\Lambda_{n}\right) H_{i n}\left(z-H_{n+1}\right)^{-1} \Lambda_{n}
\end{gathered}
$$

(это тождество доказывается так же, как и аналогичное тождество для несколько иного проекционного оператора в $\left[{ }^{6}\right]$, с. 65$)$. Учитывая, что

$$
G_{\alpha \beta}^{n}(z)=g_{\alpha \beta}^{n}(z)+\sum_{\gamma}\left\langle n, \alpha\left|\left(z-H_{n}\right)^{-1} \Lambda_{n} H_{i n}\right| n, \gamma\right\rangle g_{\gamma \beta}^{n}(z),
$$

и принимая во внимание указанное тождество, окончательно находим

$$
\sum_{\gamma} G_{\alpha \gamma}^{n}(z)\left[z \delta_{\gamma \beta}-E_{\gamma \beta}^{n}-\sum_{\delta, \varepsilon} V_{\gamma \varepsilon}^{n+1} G_{\varepsilon \delta}^{n+1} V_{\beta \delta}^{n+1}\right]=\delta_{\alpha \beta} .
$$


Формула (2) и есть треб́уемое рекуррентное соотношение МРМ, выражающее $\Phi \Gamma n$-го уровня через $Ф \Gamma n+1$-го уровня. В частном случае, когда каждому $n$ отвечает лишь один вектор $|n, 1\rangle$, эта формула сводится к рекуррентному соотношению РМ и приводит к представлению ФГ в виде цепной дроби.

Размер матриц $G^{n}$ обычно растет с ростом $n$. Поэтому при больших $n$ для решения системы (2) может оказаться выгодным использовать метод итераций, следующий из формулы эквивалентной (2):

$$
G_{\alpha \beta}^{n}(z)=g_{\alpha \beta}^{n}(z)+\sum_{\gamma, \delta, \varepsilon, \zeta} G_{\alpha \nu}^{n}(z) V_{\gamma \delta}^{n+1} G_{\delta \varepsilon}^{n+1}(z) V_{\zeta \varepsilon}^{n+1} g_{\zeta \beta}^{n}(z) \text {. }
$$

Заметим, что в случае $E_{\alpha \beta}^{n}=E_{\alpha \alpha}^{n} \delta_{\alpha \beta}$ и для каждого $\alpha$ в линейную комбинацию $H|n, \alpha\rangle$ входят определенные, не встречающиеся в других таких комбинациях векторы $|n+1, \beta(\alpha)\rangle$, решением (2) и (2') является

$$
G_{\alpha \beta}^{n}(z)=\delta_{\alpha \beta}\left[z-E_{\alpha \alpha}^{n}-\sum_{\gamma}\left|V_{\alpha \gamma}^{n+1}\right|^{2} G_{\gamma \gamma}^{n+1}(z)\right]^{-1},
$$

т. е. ФГ представляется в виде ветвящейся цепной дроби. Можно ожидать, что в случае, когда указанные условия не выполняются, а отклонения в гамильтониане от требуемого вида малы, выражение (3) может служить хорошим начальным приближением для $G^{n}$ в отмечавшейся итерационной схеме. Для максимального из принимаемых во внимание значений $n=\bar{n}$ можно положить $G_{\alpha \beta}^{\bar{n}}(z)=\delta_{\alpha \beta}\left(z-E_{\alpha \alpha}^{\bar{n}}\right)^{-1}$.

Число полюсов в $G_{11}{ }^{\circ}(z)$, вычисленной с помощью MPM, несколько меньше, но при удачном выборе базиса - порядка числа учитываемых векторов $|n, \alpha\rangle$. В РM без экстраполяции и при том же ограничении базиса число полюсов равно $\bar{n}+1$. Как легко видеть, первое число может существенно превосходить второе. Действительно, пусть для каждого $n$ векторов $|n+1, \alpha\rangle$ в $v$ раз больше, чем $|n, \alpha\rangle$, тогда число полюсов, получаемое в МРM, порядка $\sum_{i=0}^{\bar{n}} v^{i}=\left(1-v^{\bar{n}+1}\right) /(1-v) \gg \bar{n}+1$ при $v>1$ и $\bar{n} \gg 1$. Таким образом, при использовании ограниченного базиса состояний MPM дает более полное описание ФГ в сравнении с PM.

В качестве примера использования приведенного выше формализма рассмотрим гамильтониан

$$
H=-\frac{B}{12} \sum_{\mathrm{m}} \sum_{\mathrm{a}}^{\prime} a_{\mathrm{m}+\mathrm{a}}^{+} a_{\mathrm{m}}+\omega \sum_{\mathrm{m}} b_{\mathrm{m}}^{+} b_{\mathrm{m}}+\sqrt{S \omega} \sum_{\mathrm{m}} a_{\mathrm{m}}^{+} a_{\mathrm{m}}\left(b_{\mathrm{m}}^{+}+b_{\mathrm{m}}\right),
$$

описывающий систему френкелевских экситонов, контактно взаимодействующих с ветвью бездисперсных оптических фононов в простой кубической решетке. $a_{\mathrm{m}}^{+}\left(b_{\mathrm{m}}^{+}\right), B, \omega$ и $S$ - оператор рождения экситона (фонона) на узле $\mathrm{m}$, ширина экситонной зоны, частота фонона и стоксов сдвиг соответственно. Штрих у знака суммы означает, что суммирование по а проводится по 6 ближайшим соседям нулевого узла. В качестве состояния $|0,1\rangle$ примем $N^{-1 / 2} \sum_{\mathrm{m}} a_{\mathrm{m}}^{+}|\mathrm{vac}\rangle$, где $|\mathrm{vac}\rangle$ вакуумное состояние экситонной и фононной подсистем, $N-$ число узлов в периодической области кристалла. В этом случае $\operatorname{Im} G_{11}^{0}(\Omega+i \gamma)$, $\gamma \rightarrow+0$, описывает спектр оптического поглощения кристалла в экситонной области при нулевой температуре $\left[{ }^{3}\right]$. В качестве базиса состояний выберем следующий:

$$
|n, \alpha\rangle=\frac{1}{\sqrt{N N_{n \alpha}}} \sum_{1} \sum_{r}\left(b_{r \mathrm{~m}_{1}+1}^{+}\right)^{v_{1}} \ldots\left(b_{r \mathrm{~m}_{\mu}+1}^{+}\right)^{v_{\mu}} a_{1}^{+}|\mathrm{vac}\rangle
$$


где набор векторов $\mathbf{m}_{i}$ и целых чисел $v_{i}$ характеризует данное состояние $|n, \alpha\rangle, r$ - операции группы симметрии $O_{h}$, в суммирование по $r$ включены лишь такие из них, которые приводят к различающимся конфигурациям фононного облака вокруг экситона. Их число, $N^{\prime}{ }_{n \alpha}$, легко находится для заданных значений $\mathbf{m}_{i}, v_{i}$. C этим числом связана

нормировочная константа $N_{n \alpha}: N_{n \alpha}=N_{n \alpha}^{\prime} \prod_{i=1}^{\mu} v_{i} !$. Выбор симметризо-

ванных комбинаций (5) в качестве базисных состояний обусловлен симметрией гамильтониана и начального состояния $|0,1\rangle$ (обратим внимание на то, что, как и $|0,1\rangle$, эти состояния отвечают нулевому волновому вектору). Как уже отмечалось, можно использовать и несимметризованный базис, например, в виде (5) без суммирования по $r$. В этом случае $N^{\prime}{ }_{n \alpha}=1$ и нет нужды вычислять эти величины, что несколько упрощает процедуру определения $E_{\alpha \beta}^{n}$ и $V_{\alpha \beta}^{n}$, однако при этом растет объем требуемой памяти ЭВМ и количесто операций при той же информации о ФГ.

Рассмотрим действие гамильтониана на состояния (5) и начнем с первого слагаемого в правой части $(4)$, обозначаемого $K$ :

$K|n, \alpha\rangle=\frac{1}{\sqrt{N N_{n \alpha}}}\left(-\frac{B}{12}\right) \sum_{1, r, \mathrm{a}}^{\prime}\left(b_{r \mathrm{~m}_{t}+1-\mathrm{a}}^{+}\right)^{v_{1}} \ldots\left(b_{r \mathrm{~m}_{\mu}+1-\mathbf{a}}^{+}\right)^{v_{\mu}} a_{1}^{+}|\mathrm{vac}\rangle$.

В (6) входят в общем случае несколько векторов типа (5), отвечающих значениям $\mathrm{m}_{i}^{\prime}=\mathrm{m}_{i}-\mathrm{a}$ и тому же набору $v_{i}$. Для определения коэффициента перед одним из них, скажем, $\left|n^{\prime}, \alpha^{\prime}\right\rangle$, заметим, что если из одного элемента суммы по $r$ при суммировании по а возникает $\chi$ компонент этого вектора, то всего этих компонент в обеих суммах $N^{\prime}{ }_{n \alpha} \varkappa$. В вектор входит $N^{\prime}{ }_{n}^{\prime} \alpha^{\prime}$ компонент. Учитывая это, а также то обстоятельство, что нормировочной константой перед вектором должна быть $N_{n^{\prime} \alpha^{\prime}}=N^{\prime} n^{\prime} \alpha^{\prime} \prod_{i=1}^{\mu} v_{i} !$, находим требуемый коэффициент: $(-B / 12) x \sqrt{N_{n \alpha}^{\prime} / N_{n^{\prime} \alpha^{\prime}}^{\prime}} n^{\prime}=n+1, \quad n-1$ либо $n$, что определяется в ходе построения. Таким образом, найденный коэффициент отвечает величинам $E_{\alpha \beta}^{n}$ или $V_{\alpha \beta}^{n}$ в (1). Аналогичным образом находим, что второе слагаемое в (4) дает вклад в величины $E_{\alpha \alpha}^{n}$ равный $\omega \sum_{i=1}^{\mu} v_{i}$ (если $K|n, \alpha\rangle$ не содержит $|n, \alpha\rangle$, то этим вкладом и определяется $\left.E_{\alpha \alpha}^{n}\right)$; третье слагаемое, действуя на вектор (5), преобразует его в вектор с дополнительным оператором $b_{\mathbf{m}_{t}+1}^{+}$, где $\mathbf{m}_{i}=0$, причем вклад этого слагаемого в $V_{\alpha \beta}^{n+1}$ равен $\sqrt{S_{\omega}\left(v_{0}+1\right)}$, где $v_{0}$ - число операторов рождения фононов в (5) с $\mathrm{m}_{i}=0$. Четвертое слагаемое в (4) можно не рассматривать, поскольку оно эрмитово сопряжено с третьим и новой информации о $V_{\alpha \beta}^{n}$ не дает. Подчеркнем, что характер указанных выше вычислений не ведет к каким-либо неустойчивостям, в отличие от РM, где получаемые в ходе расчета вектора базиса Ланцоша с ростом $n$ теряют взаимную ортогональность и приходится принимать специальные меры по ее восстановлению [ $\left.{ }^{5}\right]$.

Руководствуясь перечисленными выше правилами легко определить константы и структуру гамильтониана (1) и вычислить ФГ. В данном случае удобно для векторов $|n, \alpha\rangle$ ввести иное обозначение $\left(\mathrm{m}_{1} \ldots \mathrm{m}_{\lambda}\right)$, где векторы $\mathrm{m}_{i}$ (тройки чисел - их декартовы координаты) отвечают одной из конфигураций фононов в симметризованной 
комбинации (5). $\quad E_{11}^{0}=-B / 6 . \quad$ В группе состояний с $n=1$ имеется лишь одно состояние - $(000), \quad V_{41}^{1}=\sqrt{S_{\omega}}, E_{11}^{1}=\omega . \quad$ В группе $n=2$ два состояния: $\quad(001), \quad V_{11}^{2}=(-B / 12) \sqrt{6}, E_{11}^{2}=\omega \quad$ и $\quad(000,000)$, $V_{12}^{2}=\sqrt{2 S_{\omega}}, E_{22}^{2}=2 \omega, E_{12}^{2}=0 . \quad$ В группе $n=3$ пять состояний: $(002)$, $V_{11}^{3}=-B / 12, E_{11}^{3}=\omega ; \quad(011), \quad V_{12}^{3}=-B / 3 \sqrt{2}, \quad E_{22}^{3}=\omega ; \quad(000,001)$, $V_{13}^{3}=\sqrt{2 S_{\omega}}, \quad E_{33}^{3}=2 \omega ; \quad(001,001), \quad V_{24}^{3}=(-B / 12) \sqrt{6}, \quad E_{44}^{3}=2 \omega ;$ $(000,000,000), \quad V_{25}^{3}=\sqrt{3 S_{\omega}}, E_{55}^{3}=3 \omega$, остальные элементы матриц $V^{3}$ и $E^{3}$ нулевые. Подобный подсчет может быть продолжен для больших $n$. При этом выясняется, что структура гамильтониана данной задачи близка к той, которая требуется для возможности использования формулы (3) для ФГ. Таким образом, по крайней мере в нулевом приближении мы избавляемся от матричных уравнений, а влияние отклонений структуры гамильтониана от требуемой для формулы (3) можно оценить с помощью упоминавшейся итерационной процедуры. По сравнению с РМ, использовавшимся для этой задачи в $\left[{ }^{3}\right]$, все вычисления оказываются значительно более простыми, не ведут к неустойчивостям и дают существенно более точное описание ФГ (ограничившись, скажем, $\bar{n}=10$, в $M$ РМ ФГ описывается несколькими тысячами $\delta$ - функций, вместо 11 в РМ).

Рассмотрим теперь случай, когда усреднение в ФГ производится по каноническому ансамблю

$$
\begin{gathered}
G_{11}^{0}(z)=i \int_{0}^{\infty} \mathrm{dt} e^{-i z t} G_{11}^{0}(t), \\
G_{11}^{0}(t)=\left\langle A_{0,1}(t) A_{0,1}^{+}\right\rangle=\operatorname{Sp}\left\{e^{-H / T} A_{0,1}(t) A_{0,1}^{+}\right\} / \operatorname{Sp}\left(e^{-H / T}\right), \\
A_{0,1}(t)=e^{i H t} A_{0,1} e^{-i H t},
\end{gathered}
$$

$A_{0,1}$ - некоторый оператор, нормированный условием $\left\langle A_{0,1} A_{0,1}^{+}\right\rangle=1$, $T$ - температура. Для вычисления таких ФГ может использоваться PM, основывающийся на модифицированном алгоритме Ланцоша аналоге обычного алгоритма Ланцоша $\left[{ }^{4,7-9}\right]$. Как будет видно из дальнейшего, MPM для этих ФГ также аналогичен обсуждавшемуся выше MPM. Коммутатор $\left[H, A_{0,1}\right]$ помимо оператора $A_{0,1}$ содержит и другие операторы, вид которых зависит от выбранного базиса операторов. Будем далее считать, что операторы базиса удовлетворяют условию (ортогональности и нормировки) $\left\langle A_{i} A_{k}^{+}\right\rangle=\delta_{i k}$. Обозначим фигурирующие в $\left[H, A_{0,1}\right]$ отличные от $A_{0,1}$ базисные векторы $A_{1, \alpha}$. В линейной комбинации $\left[H, A_{1, \alpha}\right]$ новые базисные векторы обозначим $A_{2, \beta}$. Продолжая эту процедуру заметим, что ввиду эрмитовости гамильтониана в линейную комбинацию $\left[H, A_{n, \alpha}\right], n>1$, входят лишь операторы $A_{n+1, \beta}, A_{n, \nu}$ и $A_{n-1, \delta}$. Действительно, поскольку по построению $\left\langle\left[H, A_{n-k, \beta}\right] A_{n, \alpha}^{+}\right\rangle=0, \quad k \geqslant 2$, то $\left\langle\left[H, A_{n, \alpha}\right] A_{n-k, \beta}^{+}\right\rangle=0 . \quad$ Таким образом, имеют место соотношения

$$
\left[H, A_{n, \alpha}\right]=\sum_{\beta}\left[V_{\alpha \beta}^{n+1} A_{n+1, \beta}+E_{\alpha \beta}^{n} A_{n, \beta}+\grave{V}_{\beta \alpha}^{n} A_{n-1, \beta}\right], \quad V^{0}=0 .
$$

Введем проекционный оператор $P_{n}: P_{n} Q=\sum_{\alpha}\left\langle Q A_{n, \alpha}^{+}\right\rangle A_{n, \alpha}$ 
пройзвольного оператора $Q$. Определим временную зависимость опе̂раторов $A_{n, \alpha}(t)$ соотношением

$$
\frac{d}{\mathrm{dt}} A_{n, \alpha}(t)=i \prod_{k=0}^{n-1}\left(1-P_{k}\right)\left[H, A_{n, \alpha}(t)\right], \quad A_{n, \alpha}(0)=A_{n, \alpha}
$$

(таким образом, $\left\langle A_{n, \alpha}(t) A_{k, \beta}^{+}\right\rangle=0$ при $\left.k<n\right), \quad G_{\alpha \beta}^{n}(t)=\left\langle A_{n, \alpha}(t) A_{n, \beta}^{+}\right\rangle$: Аналогично $\left[{ }^{7,8}\right]$ можно показать, что

$$
A_{n, \alpha}(t)=\sum_{\beta} G_{\alpha \beta}^{n}(t) A_{n, \beta}+i \int_{0}^{\infty} d s \sum_{\beta, \gamma} G_{\alpha \beta}^{n}(s) V_{\beta \gamma}^{n+1} A_{n+1, \gamma}(t-s) .
$$

Используя это равенство, находим

$$
\frac{d}{\mathrm{dt}} G_{\alpha \beta}^{n}(t)=i \sum_{\gamma} G_{\alpha \gamma}^{n}(t) E_{\nu \beta}^{n}-\sum_{\gamma, \delta, \varepsilon} V_{\beta \delta}^{n+1} V_{\gamma \varepsilon}^{n+1} \int_{0}^{t} d s G_{\alpha \gamma}^{n}(s) G_{\varepsilon \delta}^{n+1}(t-s),
$$

переходя затем к лаплас-образам, $\quad G_{\alpha \beta}^{n}(\mathrm{z})=i \int_{0}^{\infty} \mathrm{dt} \exp (-i z t) G_{\alpha \beta}^{n}(t)$, получаем (2). Все сказанное о МРМ относится, разумеется, и к этому случаю.

\section{ЛИ ТЕРАТ У РА}

1. Haydock, R. Solid State Phys., 35, 216-294 (1980); Kelly, M. J. Solid State Phys., $35, \quad 295-383(1980)$.

2. The Recursion Method and Its Applications (eds D. G. Pettifor, D. L. Weaire).

Berlin-Heidelberg-New York-Tokyo, Springer Verlag, 1985.

3. Sherman, A. V. Phys. status solidi (b), 135, № 2, 697-705' (1986).

4. Шерман А. Изв. АН ЭССР. Физ. Матем., 37, № 3, 280-293 (1988).

5. Парлетт Б. Симметричная проблема собственных значений. М., «Мир» 1983.

6. Кролль Н. В кн.: Квантовая оптика и квантовая радиофизика. М., «Мир», 1966, $9-89$.

7. Sherman, A. V. J. Phys. A, 20, № 3, 569-576 (1987).

8. Nagano, K., Karasudani, T., Okamoto, H., Mori, H. Progr. Theor. Phys., 63, № 6, 1904-1916 (1980).

9. Lee, M. H. Phys. Rev. B, 26, № 5, 2547-2551 (1982).

\section{Институт физики \\ Академии наук Эстонской ССР \\ Поступила в редакцию $21 /$ IX 1987}

\section{A. SERMAN}

\section{GREENI FUNKTSIOONIDE ARVUTAMISE MAATRIKSREKURSIIVMEETODIST}

On käsitletud maatriksrekursiivmeetodi varianti, millel kindla ülesannete klassi jaoks on mõningad eelised, vōrreldes hariliku rekursiivmeetodiga. Muu hulgas annab see variant sama arvu baasiolekute korral täielikumat informatsiooni Greeni funktsioonide kohta ja arvutused ei vii Lanczosi algoritmis tekkivatele ebastabiilsustele. Näitena on vaadeldud eksiton-foonon-süsteemi Greeni funktsiooni.

\section{A. SHERMAN}

\section{ON THE MATRIX RECURSION METHOD FOR CALCULATING GREEN'S FUNCTIONS}

A version of the matrix recursion method possessing some advantages in comparison with the ordinary recursion method for a definite class of problems is discussed. In particular, at the same number of basis states this version gives a more complete information about the Green's function and calculations do not lead to instabilities of the type of these arising in the Lanczos algorithm. As an example, the Green's function of the exciton-phonon system is considered. 